\title{
Effectiveness of Colorectal Cancer Screening Promotion Using E-Media Decision Aids: A Systematic Review and Meta-Analysis
}

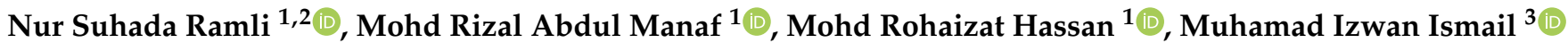 \\ and Azmawati Mohammed Nawi ${ }^{1, *(D)}$ \\ 1 Department of Community Health, Faculty of Medicine, Universiti Kebangsaan Malaysia, Jalan Yaakob Latif, \\ Cheras, Kuala Lumpur 56000, Malaysia; nursuhadaramli99@gmail.com (N.S.R.); \\ mrizal@ppukm.ukm.edu.my (M.R.A.M.); rohaizat@ppukm.ukm.edu.my (M.R.H.) \\ 2 Ministry of Health, Malaysia, Federal Government Administrative Centre, Putrajaya 62514, Malaysia \\ 3 Department of Surgery, Hospital Sultanah Aminah, Jalan Persiaran Abu Bakar Sultan, \\ Johor Bahru 80100, Malaysia; izwanismail99@gmail.com \\ * Correspondence: azmawati@ppukm.ukm.edu.my
}

check for updates

Citation: Ramli, N.S.; Manaf, M.R.A.; Hassan, M.R.; Ismail, M.I.;

Mohammed Nawi, A. Effectiveness of

Colorectal Cancer Screening

Promotion Using E-Media Decision

Aids: A Systematic Review and Meta-Analysis. Int. J. Environ. Res. Public Health 2021, 18, 8190. https:/ / doi.org/10.3390/ijerph18158190

Academic Editors: Ramune Jacobsen and Mina Nicole Händel

Received: 24 June 2021

Accepted: 30 July 2021

Published: 2 August 2021

Publisher's Note: MDPI stays neutral with regard to jurisdictional claims in published maps and institutional affiliations.

Copyright: (c) 2021 by the authors. Licensee MDPI, Basel, Switzerland. This article is an open access article distributed under the terms and conditions of the Creative Commons Attribution (CC BY) license (https:/ / creativecommons.org/licenses/by/ $4.0 /)$.
Abstract: Colorectal cancer (CRC)-screening reduces mortality, yet remains underutilized. The use of electronic media (e-media) decision aids improves saliency and fosters informed decision-making. This systematic review aimed to determine the effectiveness of CRC-screening promotion, using e-media decision aids in primary healthcare (PHC) settings. Three databases (MEDLINE, Web of Science, and the Cochrane Library) were searched for eligible studies. Studies that evaluated e-media decision aids compared to usual care or other conditions were selected. Quality was assessed by using Cochrane tools. Their effectiveness was measured by CRC-screening completion rates, and meta-analysis was conducted to calculate the pooled estimates. Ten studies involving 9393 patients were included in this review. Follow-up durations spanned 3-24 months. The two types of decision-aid interventions used were videos and interactive multimedia programs, with durations of 6-15 min. Data from nine feasible studies with low or some risk of bias were synthesized for meta-analysis. A random-effects model revealed that CRC-screening promotion using e-media decision aids were almost twice as likely to have screening completion than their comparisons (OR 1.62, 95\% CI: 1.03-2.62, $p<0.05)$. CRC-screening promotion through e-media has great potential for increasing screening participation in PHC settings. Thus, its development should be prioritized, and it should be integrated into existing programs.

Keywords: colorectal cancer screening; effectiveness; electronic media; decision aids; systematic review; meta-analysis

\section{Introduction}

Colorectal cancer $(\mathrm{CRC})$ remains the third most common malignancy worldwide, and it was ranked the second leading cause of cancer deaths in 2018 [1]. Recent data from the World Health Organization confirm that CRC is the third most common cancer in men, after lung and prostate cancer, and the second most common cancer in women, following breast cancer [2]. The incidence of CRC has continued to increase in countries across various geographical areas and development levels, and in younger populations [3]. The constellation of factors associated with westernization, such as obesity, physical inactivity, high consumption of red meat, excessive alcohol intake, and smoking, have tremendously contributed towards the high CRC incidence in economically transitioning countries [4]. Further, the economic burden of CRC is substantial and is likely to increase over time, owing to its rising incidence trend, with most patients being diagnosed at late stages [5-7].

Screening average-risk adults (age 50 to 75 years) for CRC contributes to reduced mortality [8,9]. Nonetheless, public awareness and the participation rates of CRC screening 
in most countries remain low [9-11]. In countries where CRC screening is offered in primary healthcare (PHC) services, not many participants receive physician recommendations for the screening [12-14].

It is therefore essential to invest in health-promotion programs that target the general population, focusing on increasing knowledge of the benefits of CRC screening and promoting healthy lifestyles as a matter of disease prevention. Moreover, as many patients with CRC have lower education levels [15,16], it is necessary to develop low-level-literacy health-promotion decision aids that can capture their attention and comprehension. In this digital era, rapid and innovative advances in electronic gadgets and internet communication have turned electronic media (e-media) into a powerful health-promotion tool with great opportunities for health behavior modification, as it is relatively more attractive, inexpensive, and more sustainable [17-20]. E-media decision aids that provide and support audiovisual programs should integrate a hybrid approach for digital video that enables "online" and "offline" participation to support more inclusive health and well-being promotion, using digital technology [21]. Such decision aids can deliver informational materials in various formats, such as e-reminders by health professionals via email or text message, tele-counselling, videos, or interactive multimedia programs. Although it is contended that many digitized health-promotion strategies focus on peoples' responsibility for their own health, some fail to recognize the social, cultural, and political dimensions of digital technology use [22]. Hence, such e-media should be developed according to existing cultural values.

This systematic literature review was aimed at assessing the effectiveness of e-media decision-aid interventions used in PHC settings, from identifying to reminding patients who have not responded to CRC screening. We considered a systematic literature review the most appropriate form of review to address the research question: how effective is e-media decision aid intervention, compared with a baseline or control group, in improving CRC screening among eligible patients in PHC settings? Thus, this review helps to identify future practice and strategies for improving CRC-screening effectiveness in PHC services.

\section{Materials and Methods}

\subsection{Protocol Registration}

The protocol for this systematic review was registered at Prospero (registration ID CRD42020220301) prior to the commencement of research to avoid duplication and minimize reporting bias.

\subsection{Database Used}

We searched the MEDLINE, Web of Science, and the Cochrane Library databases, because they were expected to contain relevant studies with numerous open-access full-text articles available online. The scope of MEDLINE is broadly defined and encompasses the areas used by health professionals engaged in basic research and clinical care, public health, health policy development, or related educational activities [23]. Web of Science contains a remarkable treasure trove of data on scientific content on a global scale that has been an indispensable resource for studying of science, technology, and knowledge [24]. The Cochrane Library comprises a collection of databases that contain different types of highquality independent evidence to inform healthcare decision-making, and concentrated reports of randomized and quasi-randomized controlled trials [25].

\subsection{Systematic Review Process}

The literature search was performed from November 2020 to December 2020, and we searched for articles published from January 2010 up to 4 December 2020. The 10-year span was selected based on the integration of e-media and the Internet of Things, an important technical domain that has grown rapidly in the past decade and has received wide attention globally from a plethora of disciplines, including medical research [26]. The articles were 
selected in accordance with the Preferred Reporting Items for Systematic Reviews and Meta-Analyses or PRISMA guideline [27] (Figure 1).
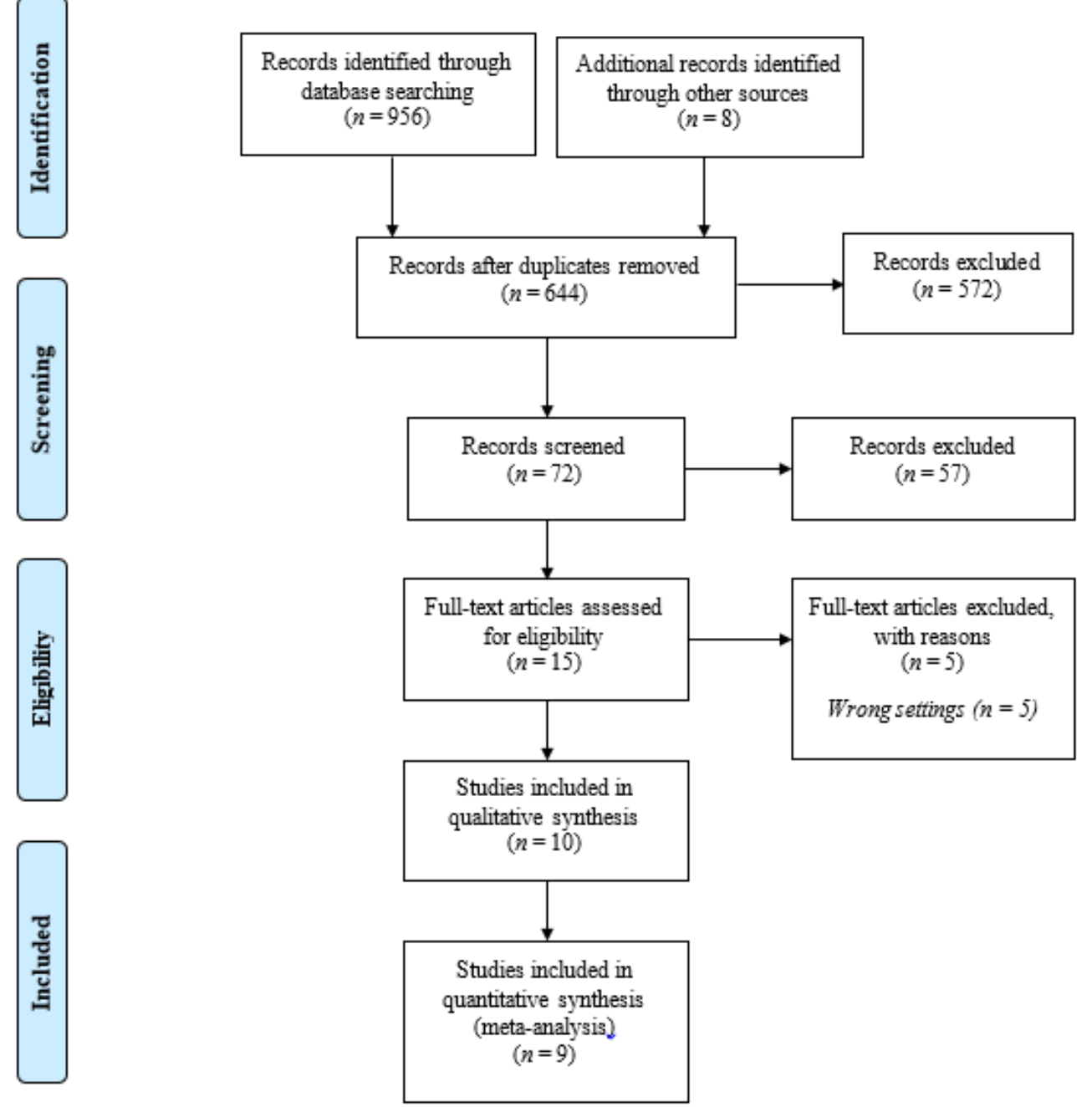

Figure 1. Study selection according to PRISMA guideline.

\subsection{Identification}

A preliminary search was conducted to identify the appropriate keywords and to determine whether this review was feasible. The keywords were verified and validated by two public health physicians at the Universiti Kebangsaan Malaysia. The medical subject heading (MeSH) keywords used were as follows: ("colorectal cancer" OR "colon cancer" OR "bowel cancer" OR "rectal cancer" OR "colorectal neoplasm" OR "colorectal carcinoma") AND ("screening" OR "detection" OR "prevention" OR "colonoscopy" OR "fecal occult blood" OR "FOBT") AND ("media" OR "mass media" OR "electronic media" OR "video") (Supplementary Figure S1).

Potential additional studies were identified through reference tracking of systematic reviews during the database search. Given the extensive nature of the three databases, we did not search unpublished reports.

\subsection{Screening}

The initial search retrieved 964 articles (Medline $=124$, Web of Science $=226$, the Cochrane Library $=203$, and reference tracking $=8$ ). The search results were imported into the EndNote reference manager, and duplicates were removed $(n=312)$. Basic information from the remaining articles was exported to a Microsoft Excel sheet. Two authors (NSR and 
MII) independently reviewed all titles, abstracts, and references generated by the original search to identify articles for potential inclusion. A total of 644 titles and abstracts were screened based on their relevance to the inclusion and exclusion criteria, resulting in the exclusion of 572 articles, leaving 72 articles to be assessed for eligibility.

\subsection{Eligibility}

The inclusion criteria were limited to randomized or quasi-experiment trials of CRC-screening promotion interventions using e-media, based on the PICO framework: (a) population-eligible patients in PHC settings; (b) intervention-CRC-screening promotional tools using e-media; (c) comparison-usual care or otherwise specified; and (d) outcome-CRC-screening completion, either fecal occult blood test (FOBT) or colonoscopy or sigmoidoscopy uptake. In addition, eligible articles had to be published in English, with open-access full-text articles available online. We used the modified Australian National Bowel Cancer Screening Program (NBCSP) Quality Framework [28] as a guideline for identifying eligible patients and for optimizing their participation at PHC centers in selected studies (Figure 2). This framework was chosen because it illustrates a similar concept of the CRC-screening clinical pathway used in other countries. Articles were excluded if they failed to meet the inclusion criteria, or assessed interventions that involved surveillance colonoscopy or follow-up after cancer treatment.

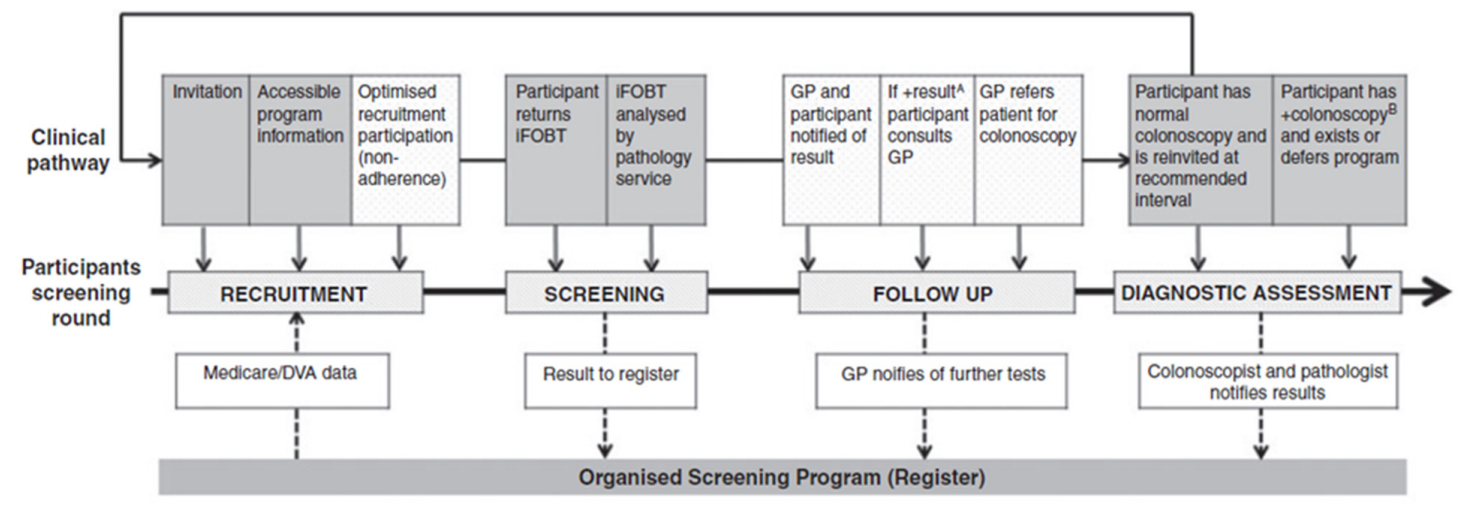

Figure 2. The modified National Bowel Cancer Screening Program Quality Framework, version 2. A A positive (+) result means that blood was detected in the completed immunochemical fecal occult blood test (iFOBT). ${ }^{B}$ A positive colonoscopy is identified by reporting one of the following: tubular adenoma, tubulovillous adenoma, villous adenoma, sessile serrated adenoma, traditional serrated adenoma, adenoma not otherwise classified, or carcinoma.

A total of 57 articles did not meet the eligibility criteria. In the next stage, two authors (NSR and AMN) assessed the full articles independently and compared the results. To increase the reliability of the study selection, all differences were reconciled by consensus. In the full assessment of the remaining 15 articles, five were excluded for falling outside the scope of this review, resulting in the selection of a final 10 articles.

\subsection{Data Extraction}

At the final stage, NSR extracted data by using a standardized Excel spreadsheet, which was revised by another three authors (AMN, MRH, and MRM). The data extracted for mapping and analysis included author, year of publication, study country, study design, participants, sample size, type of intervention including content and duration, comparison, main outcome measures, and results. The outcome measures and operational definitions used for describing CRC-screening promotion effectiveness are defined in Table 1. The effectiveness of the primary outcome of interest (CRC-screening completion) was categorized into either effective, i.e., higher CRC-screening completion rate with statistical significance; null, i.e., higher or lower CRC-screening completion rate without statistical significance; or not effective, i.e., lower CRC-screening completion rate with statistical significance, and was reported as our final outcome summary. 
Table 1. Outcome measures and operational definitions used.

\begin{tabular}{ccc}
\hline Outcome Measures & Operational Definition \\
\hline Primary & SRC-screening completion rate & $\begin{array}{c}\text { Completion rate or participation rate or adherence rate to either FOBT } \\
\text { or colonoscopy or sigmoidoscopy uptake. }\end{array}$ \\
\cline { 2 - 3 } Secondary & CRC-screening awareness and belief & $\begin{array}{c}\text { Spoiled FOBT kits are samples which cannot be analyzed for one reason } \\
\text { or another (e.g., no date on the specimen). }\end{array}$ \\
\cline { 2 - 3 } & Ability to state CRC-screening test preference & $\begin{array}{c}\text { Awareness score was calculated by summing the points earned for all } \\
\text { awareness items. A scale assessing influence of religious beliefs on } \\
\text { medical-decision-making, such as CRC screening, was used. }\end{array}$ \\
\hline Readiness to receive CRC screening & $\begin{array}{c}\text { Assessment on the post-program survey by asking patients which } \\
\text { CRC-screening test they would want if all tests were free. }\end{array}$ \\
\hline Ordered CRC-screening tests & $\begin{array}{c}\text { Determined by comparing patients' readiness stage after the } \\
\text { intervention program to their baseline stage. }\end{array}$ \\
\hline
\end{tabular}

Abbreviation: $\mathrm{CRC}=$ colorectal cancer, $\mathrm{FOBT}=$ fecal occult blood test.

\subsection{Risk of Bias Assessment}

We assessed study quality by using the Cochrane risk-of-bias tool for randomized trials (RoB 2) and the risk-of-bias tool for cluster randomized trials (RoB 2 CRT) because they are the most commonly used tool for randomized trials [29]. Bias was assessed in five distinct domains, where answers were required for one or more signaling questions. These answers led to evaluations of "low risk of bias", "some concerns", or "high risk of bias". The evaluations within each domain led to an overall risk of bias evaluations for the result being assessed, which should enable the stratification of meta-analyses according to the risk of bias.

\subsection{Data Analysis}

All statistical analyses were performed by using Review Manager 5.4.1 (Cochrane, London, UK) [30]. We included "low risk of bias" and "some concerns" studies in the meta-analysis. Comparable data from studies were pooled by using forest plots. The odd ratios (ORs) with 95\% confidence intervals (CIs) for dichotomous data were used as the effect measure and were reported as the primary outcome. Inter-study heterogeneity was assessed by using the $I^{2}$ statistic for each pooled estimate. We used a random-effects model for heterogeneity $(p<0.05)$. Due to the possibility of clinical homogeneity, we performed subgroup analysis on the type of e-media decision aid used and the target population. The robustness of the results was evaluated with sensitivity analysis by excluding individual studies from each forest plot. Publication bias was assessed by using funnel plots.

\section{Results}

\subsection{Characteristic of Studies}

A total of 10 studies were included in this review. Eight studies were conducted in the United States [31-38], while the remaining two took place in New Zealand [39] and Iran [40]. Eight studies were randomized controlled trials [31-33,35,37-40]; another two had quasi-experimental designs $[34,36]$.

Four studies followed their patients for a minimum of 3 months [32,35,38,39]; one for 4 months [40]; two for 6 months [33,37]; and one each for 12 months [36], 14 months [31], and the maximum duration of 24 months [34]. The majority of studies reported interventions targeting eligible patients [31-38,40]; one study targeted non-adherence patients [39]. More than half of the included studies focused on improving the screening participation of specific vulnerable populations that tend to be under-screened or never screened [32-36,38,39].

In total, 9393 patients were had follow-ups. The sample sizes ranged from 65 [38] to 5271 [39], with most studies taking place in a multi-clinic setting [31-36,40]. Table 2 provides an overview of the characteristics of the included studies. 
Table 2. Table of evidence from all ten studies in this review $(N=10)$.

\begin{tabular}{|c|c|c|c|c|c|c|c|c|c|}
\hline No. & Author (Year) & Country & $\begin{array}{l}\text { Study Design } \\
\text { (Follow-Up) }\end{array}$ & $\begin{array}{l}\text { Participants } \\
\text { (Sample Size) }\end{array}$ & $\begin{array}{c}\text { Type of e-Media } \\
\text { Decision-Aid Used }\end{array}$ & $\begin{array}{l}\text { E-media Decision-Aid Content } \\
\text { (Duration) }\end{array}$ & Comparison & $\begin{array}{l}\text { Main Outcome } \\
\text { Measures }\end{array}$ & Results \\
\hline 1 & $\begin{array}{l}\text { Walsh et al. } \\
(2020)\end{array}$ & $\begin{array}{l}\text { San Francisco, } \\
\text { United States }\end{array}$ & $\begin{array}{l}\text { RCT } \\
\text { (14 months) }\end{array}$ & $\begin{array}{l}\text { Patients with } \\
\text { upcoming follow-up } \\
\text { or preventive } \\
\text { appointments } \\
\text { (508, } 6 \text { clinics) }\end{array}$ & $\begin{array}{l}\text { Interactive } \\
\text { multimedia program } \\
\text { ("Video Doctor" and } \\
\text { "Provider Alert" } \\
\text { paper, named } \\
\text { "PreView" } \\
\text { (Preventive Video } \\
\text { Education in } \\
\text { Waiting Areas)) }\end{array}$ & $\begin{array}{l}\text { - Series of questions (health } \\
\text { assessment, prior cancer } \\
\text { screening, and readiness to } \\
\text { change cancer screening } \\
\text { behavior) } \\
\text { - Stage of change assessment } \\
\text { "Video Doctor" } \\
\text { conversation between a } \\
\text { patient and a physician } \\
\text { - Participants' receipt of } \\
\text { "Provider Alert" } \\
\text { individualized messages } \\
\text { (Not mentioned) }\end{array}$ & $\begin{array}{l}\text { Video about } \\
\text { healthy lifestyle }\end{array}$ & $\begin{array}{ll}\text { - } & { }^{\mathrm{c}} \mathrm{CRC}- \\
& \text { screening } \\
& \text { completion }^{\mathrm{f}}\end{array}$ & $\begin{array}{l}\text { CRC-screening completion } \\
\text { rate was higher in the } \\
\text { intervention group than in } \\
\text { the control group } \\
(78.5 \% \text { vs. } 77.0 \%)^{* *} \text {. }\end{array}$ \\
\hline 2 & $\begin{array}{l}\text { Bartholomew } \\
\text { et al. (2019) }\end{array}$ & $\begin{array}{l}\text { Auckland, } \\
\text { New Zealand }\end{array}$ & $\begin{array}{l}\text { RCT } \\
\text { (3 months) }\end{array}$ & $\begin{array}{l}\text { Māori and Pacific } \\
\text { residents, } \\
\text { non-adherent to } \\
\text { initial screening } \\
\text { invitation } \\
\text { (5271, clinic numbers } \\
\text { not mentioned) }\end{array}$ & $\begin{array}{l}\text { Video (DVD and a } \\
\text { reminder letter) }\end{array}$ & $\begin{array}{l}\text { - The importance of CRC } \\
\text { screening } \\
\text { - The ease of the test } \\
\text { - The nature of return of } \\
\text { results } \\
\text { - Participants' positive } \\
\text { experiences of the diagnostic } \\
\text { follow-up test } \\
\text { (6 min) }\end{array}$ & $\begin{array}{l}{ }^{\mathrm{d}} \text { Usual } \\
\text { reminder letter }\end{array}$ & $\begin{array}{ll}- & { }^{a} \text { CRC- } \\
\text { screening } \\
\text { participation } \\
\text { e Spoiled } \\
\text { FOBT } \\
\text { kit return }\end{array}$ & $\begin{array}{l}\text { CRC-screening participation } \\
\text { rate was lower in the } \\
\text { intervention groups than in } \\
\text { the control groups, for both } \\
\text { ethnic groups (13.6\% vs. } \\
25.9 \% \text { in Māori and } \\
10.1 \% \text { vs. } 18.4 \% \text { in Pacific) }{ }^{*} \text {. } \\
\text { Spoiled kit rates were lower } \\
\text { in the intervention groups } \\
\text { than in the control groups } \\
(12.4 \% \text { vs. } 33.1 \% \text { in Māori } \\
\text { and } 21.9 \% \text { vs. } 42.1 \% \\
\text { in Pacific) }\end{array}$ \\
\hline 3 & $\begin{array}{l}\text { Gwede et al. } \\
(2019)\end{array}$ & $\begin{array}{l}\text { Florida, } \\
\text { United States }\end{array}$ & $\begin{array}{l}\text { RCT } \\
\text { (3 months) }\end{array}$ & $\begin{array}{l}\text { Latinos population } \\
\text { ( } 76,2 \text { clinics })\end{array}$ & $\begin{array}{l}\text { Video (DVD with } \\
\text { fotonovela booklet } \\
\text { and FIT kit, called } \\
\text { "LCARES" (Latinos } \\
\text { CRC Awareness, } \\
\text { Research, Education, } \\
\text { and Screening)) }\end{array}$ & $\begin{array}{l}\text { - Constructs of a preventive } \\
\text { health model for CRC } \\
\text { screening, e.g., salience and } \\
\text { coherence, cancer worry and } \\
\text { self-efficacy } \\
\text { - } \quad \text { Culturally tailored } \\
\text { (Not mentioned) }\end{array}$ & $\begin{array}{l}\text { Booklet about } \\
\text { CRC-screening } \\
\text { promotion in } \\
\text { Spanish and a FIT kit }\end{array}$ & $\begin{array}{ll}\text { - } & \text { a CRC- } \\
\text { screening } \\
\text { completion } \\
\text { CRC screening } \\
\text { awareness } \\
\text { and beliefs }\end{array}$ & $\begin{array}{l}\text { CRC-screening completion } \\
\text { rate was higher in the } \\
\text { intervention group than in } \\
\text { the control group } \\
(90 \% \text { vs. } 83 \%)^{* *} \text {. } \\
\text { The intervention group was } \\
\text { associated with greater } \\
\text { increases in CRC* } \\
\text { awareness and * } \\
\text { susceptibility, * cancer worry } \\
\text { increased more in the } \\
\text { comparison group. }\end{array}$ \\
\hline
\end{tabular}


Table 2. Cont.

\begin{tabular}{|c|c|c|c|c|c|c|c|c|c|}
\hline No. & Author (Year) & Country & $\begin{array}{l}\text { Study Design } \\
\text { (Follow-Up) }\end{array}$ & $\begin{array}{l}\text { Participants } \\
\text { (Sample Size) }\end{array}$ & $\begin{array}{c}\text { Type of e-Media } \\
\text { Decision-Aid Used }\end{array}$ & $\begin{array}{c}\text { E-media Decision-Aid Content } \\
\text { (Duration) }\end{array}$ & Comparison & $\begin{array}{l}\text { Main Outcome } \\
\text { Measures }\end{array}$ & Results \\
\hline 4 & $\begin{array}{l}\text { Besharati et al. } \\
\text { (2017) }\end{array}$ & $\begin{array}{l}\text { Hamadan City, } \\
\text { Iran }\end{array}$ & $\begin{array}{l}\text { Cluster RCT } \\
\text { (4 months) }\end{array}$ & $\begin{array}{l}\text { Iranian adults } \\
\text { ( } 248,8 \text { clinics })\end{array}$ & $\begin{array}{l}\text { Video ((educational } \\
\text { package consists of } \\
\text { video, discussion, } \\
\text { role play, a reminder } \\
\text { pack of postcards, } \\
\text { and pamphlet). } \\
\text { Group 1: } \\
\text { Educational package } \\
\text { and free FOBT; } \\
\text { Group 2: only } \\
\text { Educational package; } \\
\text { Group 3: free FOBT) }\end{array}$ & 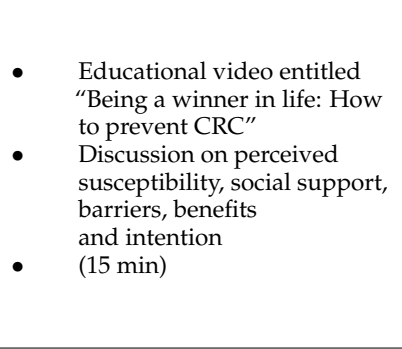 & $\begin{array}{l}\text { Usual care given } \\
\text { with survey about } \\
\text { determinants of } \\
\text { CRC-screening } \\
\text { behavior }\end{array}$ & $\begin{array}{ll}\text { - } \quad{ }^{\text {a }} \text { CRC- } \\
\text { screening } \\
\text { completion }\end{array}$ & $\begin{array}{l}\text { CRC-screening completion } \\
\text { rates were higher in the } \\
\text { three intervention groups } \\
\text { than in the control group } \\
(87.1 \% \text {, } 1.3 \% \text {, and } 54.8 \text { in } \\
\text { Group 1, Group 2, and } \\
\text { Group 3, respectively, } \\
\text { vs. } 1.6 \%)^{*} \text {. }\end{array}$ \\
\hline 5 & $\begin{array}{l}\text { Reuland et al. } \\
\text { (2017) }\end{array}$ & $\begin{array}{l}\text { North Carolina } \\
\text { and New } \\
\text { Mexico, } \\
\text { United States }\end{array}$ & $\begin{array}{l}\text { RCT } \\
\text { (6 months) }\end{array}$ & $\begin{array}{l}\text { Vulnerable low } \\
\text { income population } \\
\text { ( } 265,2 \text { clinics) }\end{array}$ & $\begin{array}{l}\text { Video (decision aid } \\
\text { and patient } \\
\text { navigation } \\
\text { (employees of the } \\
\text { clinic or its affiliated } \\
\text { health system)) }\end{array}$ & $\begin{array}{ll}\text { - } & \text { Importance of CRC } \\
& \text { screening } \\
- & \text { Review screening } \\
\text { - } & \text { test options } \\
\text { Selection of a brochure of } \\
\text { their CRC-screening } \\
\text { readiness } \\
\text { - } & (15 \mathrm{~min}) \\
\end{array}$ & $\begin{array}{l}\text { Video on food safety } \\
\text { and }^{\mathrm{d}} \text { usual care }\end{array}$ & $\begin{array}{ll}-\quad & { }^{\mathrm{b}} \text { CRC- } \\
\text { screening } \\
\text { completion }\end{array}$ & $\begin{array}{l}\text { - CRC-screening completion } \\
\text { rate was higher in } \\
\text { intervention group than in } \\
\text { the control group } \\
(68 \% \text { vs. } 27 \%)^{*} \text {. }\end{array}$ \\
\hline 6 & $\begin{array}{l}\text { Larkey et al. } \\
(2015)\end{array}$ & $\begin{array}{l}\text { Arizona, } \\
\text { United States }\end{array}$ & $\begin{array}{l}\text { RCT } \\
\text { (3 months) }\end{array}$ & $\begin{array}{l}\text { Low-income patients } \\
\text { ( } 545,15 \text { clinics) }\end{array}$ & Video & $\begin{array}{ll}- & \text { A drama about “Papa” } \\
& \text { receiving CRC screening } \\
\text { - } & \text { Information on risk } \\
\text { - } & \text { Reflecting elements for } \\
\text { behavior change in } \\
\text { cultural elements } \\
\text { - } \quad \begin{array}{l}\text { Creating dramatic tension- } \\
\text { what will Papa's test } \\
\text { results be? } \\
\text { - } 7 \mathrm{~min})\end{array}\end{array}$ & $\begin{array}{l}\text { Usual care given } \\
\text { with instrument } \\
\text { estimating level of } \\
\text { personal cancer risk } \\
\text { based on the } \\
\text { Harvard Cancer } \\
\text { Risk Index }\end{array}$ & $\begin{array}{ll}- & { }^{\mathrm{c}} \mathrm{CRC}- \\
& \text { screening } \\
& \text { adherence }\end{array}$ & $\begin{array}{l}\text { - CRC-screening adherence } \\
\text { rate was lower in the } \\
\text { intervention group than in } \\
\text { the control group } \\
(37 \% \text { vs. } 42 \%)^{* *} \text {. }\end{array}$ \\
\hline
\end{tabular}


Table 2. Cont.

\begin{tabular}{|c|c|c|c|c|c|c|c|c|c|}
\hline No. & Author (Year) & Country & $\begin{array}{l}\text { Study Design } \\
\text { (Follow-Up) }\end{array}$ & $\begin{array}{l}\text { Participants } \\
\text { (Sample Size) }\end{array}$ & $\begin{array}{c}\text { Type of e-Media } \\
\text { Decision-Aid Used }\end{array}$ & $\begin{array}{l}\text { E-media Decision-Aid Content } \\
\text { (Duration) }\end{array}$ & Comparison & $\begin{array}{l}\text { Main Outcome } \\
\text { Measures }\end{array}$ & Results \\
\hline 7 & Tu et al. (2014) & $\begin{array}{l}\text { Washington, } \\
\text { United States }\end{array}$ & $\begin{array}{l}\text { Quasi } \\
\text { experiment } \\
\text { (24 months) }\end{array}$ & $\begin{array}{l}\text { Vietnamese } \\
\text { immigrants } \\
\text { (1260, } 2 \text { clinics) }\end{array}$ & $\begin{array}{l}\text { Video ((DVD) and } \\
\text { a pamphlet) }\end{array}$ & $\begin{array}{ll}\text { - } & \text { Motivational education } \\
\text { promoting on CRC } \\
\text { screening } \\
\text { - } \quad \text { Culturally tailored } \\
\text { (Not mentioned) }\end{array}$ & $\begin{array}{l}\text { Usual care (FOBT } \\
\text { ordered by primary } \\
\text { care providers) }\end{array}$ & $\begin{array}{ll}\text { - } \quad{ }^{\mathrm{c}} \mathrm{CRC}- \\
& \text { screening } \\
& \text { adherence }\end{array}$ & $\begin{array}{l}\text { CRC-screening adherence } \\
\text { was higher in the } \\
\text { intervention group than in } \\
\text { the control clinic } \\
\text { (45\% vs. 38\%, g AOR } 1.42 ; \\
95 \% \text { CI 0.95-2.15)**. } \\
\text { Among those who were } \\
\text { non-adherent at baseline, } \\
\text { overall CRC-screening } \\
\text { adherence was higher in the } \\
\text { intervention group than in } \\
\text { the control group } \\
(47.3 \% \text { vs. } 34.5 \% \text {, } \\
\mathrm{g} \text { AOR } 1.70 ; \\
95 \% \text { CI } 1.05-2.75)^{*} \text {. }\end{array}$ \\
\hline 8 & $\begin{array}{l}\text { Davis et al. } \\
\text { (2013) }\end{array}$ & $\begin{array}{l}\text { Louisiana, } \\
\text { United States }\end{array}$ & $\begin{array}{l}\text { Quasi } \\
\text { experiment } \\
\text { (12 months) }\end{array}$ & $\begin{array}{l}\text { Low-income, } \\
\text { uninsured patients } \\
\text { in predominantly } \\
\text { rural areas } \\
\text { ( } 961,8 \text { clinics) }\end{array}$ & $\begin{array}{l}\text { Video (Educational } \\
\text { strategy (enhanced } \\
\text { usual care, pamphlet, } \\
\text { video, and FOBT } \\
\text { instructions). } \\
\text { Group 1: } \\
\text { Educational strategy; } \\
\text { Group 2: } \\
\text { Educational strategy } \\
\text { and nurse support to } \\
\text { encourage } \\
\text { CRC-screening } \\
\text { completion) }\end{array}$ & $\begin{array}{l}\text { - Patients discussing on } \\
\text { barriers and facilitators to } \\
\text { screening and a physician } \\
\text { making a recommendation } \\
\text { while showing key steps in } \\
\text { FOBT completion } \\
\text { - Not mentioned) }\end{array}$ & $\begin{array}{l}\text { Enhanced usual care } \\
\text { (recommendation for } \\
\text { CRC screening and } \\
\text { FOBT kit) }\end{array}$ & $\begin{array}{l}\text { - } \quad{ }^{\text {a CRC- }} \\
\text { screening } \\
\text { completion }\end{array}$ & 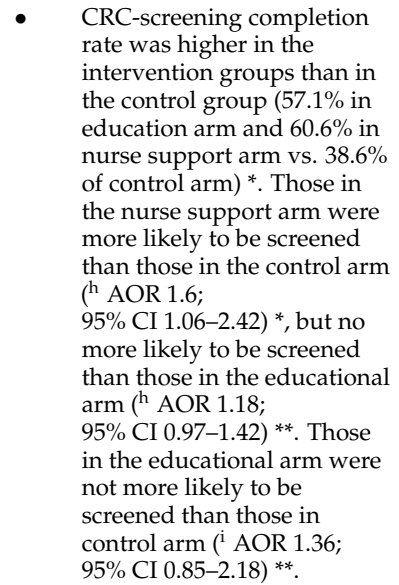 \\
\hline
\end{tabular}


Table 2. Cont

\begin{tabular}{|c|c|c|c|c|c|c|c|c|c|}
\hline No. & Author (Year) & Country & $\begin{array}{l}\text { Study Design } \\
\text { (Follow-Up) }\end{array}$ & $\begin{array}{l}\text { Participants } \\
\text { (Sample Size) }\end{array}$ & $\begin{array}{c}\text { Type of e-Media } \\
\text { Decision-Aid Used }\end{array}$ & $\begin{array}{l}\text { E-media Decision-Aid Content } \\
\text { (Duration) }\end{array}$ & Comparison & $\begin{array}{l}\text { Main Outcome } \\
\text { Measures }\end{array}$ & Results \\
\hline 9 & $\begin{array}{l}\text { Miller et al. } \\
\text { (2011) }\end{array}$ & $\begin{array}{l}\text { North Carolina, } \\
\text { United States }\end{array}$ & $\begin{array}{l}\text { RCT } \\
\text { (6 months) }\end{array}$ & $\begin{array}{l}\text { Patients scheduled } \\
\text { for routine visits } \\
\text { ( } 264,1 \text { clinic) }\end{array}$ & $\begin{array}{l}\text { Interactive } \\
\text { multimedia } \\
\text { web-based program } \\
\text { (named "CHOICE" } \\
\text { (Communicating } \\
\text { Health Options } \\
\text { through Interactive } \\
\text { Computer } \\
\text { Education, } \\
\text { version 6.0 W)) }\end{array}$ & $\begin{array}{ll}- & \text { Overview of CRC screening } \\
& \text { Education options (to learn } \\
\text { about a specific test, view } & \text { tests comparison, or end } \\
\text { the program) } & \text { Choice of screening decision } \\
-\quad & \text { A corresponding handout } \\
\text { Encouragement for } \\
\text { screening decision } \\
\text { discussion with } \\
\text { healthcare providers } \\
\text { (6.3 min overview) }\end{array}$ & $\begin{array}{l}\text { Interactive } \\
\text { web-based program } \\
\text { about prescription } \\
\text { drug refills } \\
\text { and safety }\end{array}$ & $\begin{array}{ll}\text { - } \quad{ }^{\mathrm{c}} \text { CRC- } \\
\text { screening } \\
\text { completion } \\
\text { Ability to state } \\
\text { screening test } \\
\text { preference, } \\
\text { readiness to } \\
\text { receive } \\
\text { screening, and } \\
\text { ordered } \\
\text { screening tests }\end{array}$ & $\begin{array}{l}\text { CRC-screening completion } \\
\text { rate was higher in the } \\
\text { intervention group than in } \\
\text { the control group } \\
\text { (19\% vs. } 14 \% \%^{4} \mathrm{AOR} 1.7 ; \\
95 \% \text { CI } 0.88-3.2)^{* *} . \\
\text { The rates of ability to state } \\
\text { screening test preference, } \\
\text { readiness to receive } \\
\text { screening, and ordered } \\
\text { screening tests were all } \\
\text { higher in the intervention } \\
\text { group than in the control } \\
\text { group }\left(84 \% \text { vs. } 55 \% \%^{*},\right. \\
52 \% \text { vs. } 20 \% * 30 \% \text { vs. } 21 \%, \\
\text { j AOR } 1.6 ; 95 \% \text { CI } 0.97-2.8)^{* *} \text {. }\end{array}$ \\
\hline 10 & $\begin{array}{l}\text { Aragones et al. } \\
(2010)\end{array}$ & $\begin{array}{l}\text { New York, } \\
\text { United States }\end{array}$ & $\begin{array}{l}\text { RCT } \\
\text { (3 months) }\end{array}$ & $\begin{array}{l}\text { Latino immigrants } \\
\text { (65,1 clinic) }\end{array}$ & $\begin{array}{l}\text { Video ((and a } \\
\text { brochure for patient) } \\
\text { with a paper-based } \\
\text { reminder for } \\
\text { their physicians) }\end{array}$ & $\begin{array}{ll}\text { - } & \text { Education about } \\
& \text { CRC-screening modalities, } \\
\text { prevention, and risk factors } \\
\text { - } \quad \text { Culturally tailored } \\
\text { - }(11 \mathrm{~min})\end{array}$ & ${ }^{\mathrm{d}}$ Usual care & $\begin{array}{ll} & { }^{\mathrm{c}} \mathrm{CRC}- \\
& \text { screening } \\
& \text { completion }\end{array}$ & $\begin{array}{l}\text { CRC-screening completion } \\
\text { rate was higher in the } \\
\text { intervention group than in } \\
\text { the control group } \\
\left(55 \% \text { vs. } 18 \%,{ }^{k} \text { AOR } 5.4 ;\right. \\
95 \% \text { CI } 1.6-18.5)^{*} \text {. }\end{array}$ \\
\hline
\end{tabular}

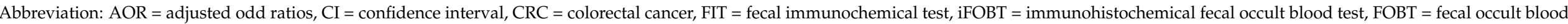

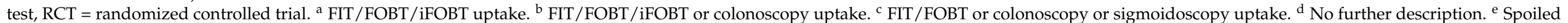

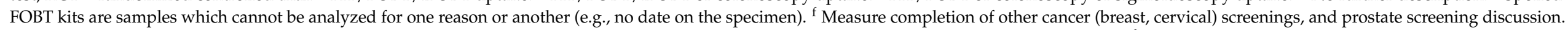

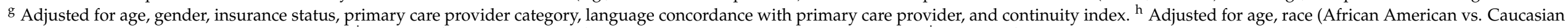

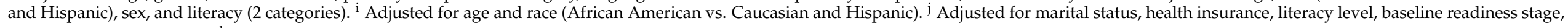

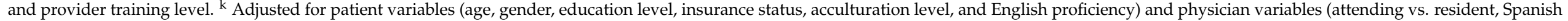
fluency). ${ }^{*} p<0.05,{ }^{* *} p>0.05$. 


\subsection{E-Media Decision Aid Intervention Type, Content and Duration Length}

The 10 studies yielded two types of decision aid interventions used for screening participation: videos, either with or without combination with other types of media tools [32-36,38-40], and interactive multimedia programs [31,37]. All contents pertained to the promotion of CRC screening and provided a basic overview of the disease and its screening options, purposely developed for easy understanding. Of the 10 included studies, four did not mention the intervention duration length; three articles reported the intervention duration length, which ranged from $6 \mathrm{~min}$ [39] to $15 \mathrm{~min}[33,40]$.

\subsection{Effectiveness of CRC-Screening Promotion Using E-Media \\ 3.3.1. Studies Included in Qualitative Synthesis}

CRC-screening completion (or participation or adherence to either FOBT or colonoscopy or sigmoidoscopy uptake) was used as a proxy for determining the effectiveness of CRCscreening promotion intervention using e-media. The final outcome summary derived from all studies was categorized accordingly (Table 3). Almost half of these studies $[33,36,38,40]$ reported effective results from their significant statistical values (Figure 3). Half of them were deemed null due to their non-significant results, despite some studies yielded higher CRC-screening rates $[31,32,35,37]$. Interestingly, the statistical analysis of one study demonstrated not effective results, showing a lower CRC-screening completion rate in the intervention group [39].

Table 3. Outcome summary for CRC-screening completion rates using e-media interventions $(N=10)$.

\begin{tabular}{|c|c|c|c|c|c|}
\hline \multirow[b]{2}{*}{ Study (Year) } & \multicolumn{4}{|c|}{ Outcome (Intervention vs. Control) } & \multirow[b]{2}{*}{$\begin{array}{l}\text { Outcome } \\
\text { Summary }\end{array}$} \\
\hline & $\begin{array}{c}\text { Higher } \\
\text { CRC-Screening } \\
\text { Completion Rate } \\
\text { with Statistical } \\
\text { Significance }\end{array}$ & $\begin{array}{c}\text { Higher } \\
\text { CRC-Screening } \\
\text { Completion Rate } \\
\text { without Statistical } \\
\text { Significance }\end{array}$ & $\begin{array}{c}\text { Lower } \\
\text { CRC-Screening } \\
\text { Completion rate } \\
\text { with Statistical } \\
\text { Significance }\end{array}$ & $\begin{array}{c}\text { Lower } \\
\text { CRC-Screening } \\
\text { Completion Rate } \\
\text { without Statistical } \\
\text { Significance }\end{array}$ & \\
\hline Walsh et al. (2020) & & $\sqrt{ }$ & & & Null \\
\hline $\begin{array}{l}\text { Bartholomew et al. } \\
\qquad(2019)\end{array}$ & & & $\sqrt{ }$ & & Not effective \\
\hline Gwede et al. (2019) & & $\sqrt{ }$ & & & Null \\
\hline $\begin{array}{l}\text { Besharati et al. } \\
\text { (2017) }\end{array}$ & $\sqrt{ }$ & & & & Effective \\
\hline $\begin{array}{l}\text { Reuland et al. } \\
\qquad(2017)\end{array}$ & $\sqrt{ }$ & & & & Effective \\
\hline Larkey et al. (2015) & & & & $\sqrt{ }$ & Null \\
\hline Tu et al. (2014) & & $\sqrt{ }$ & & & Null \\
\hline Davis et al. (2013) & $\sqrt{ }$ & & & & Effective \\
\hline Miller et al. (2011) & & $\sqrt{ }$ & & & Null \\
\hline $\begin{array}{l}\text { Aragones et al. } \\
(2010)\end{array}$ & $\sqrt{ }$ & & & & Effective \\
\hline
\end{tabular}




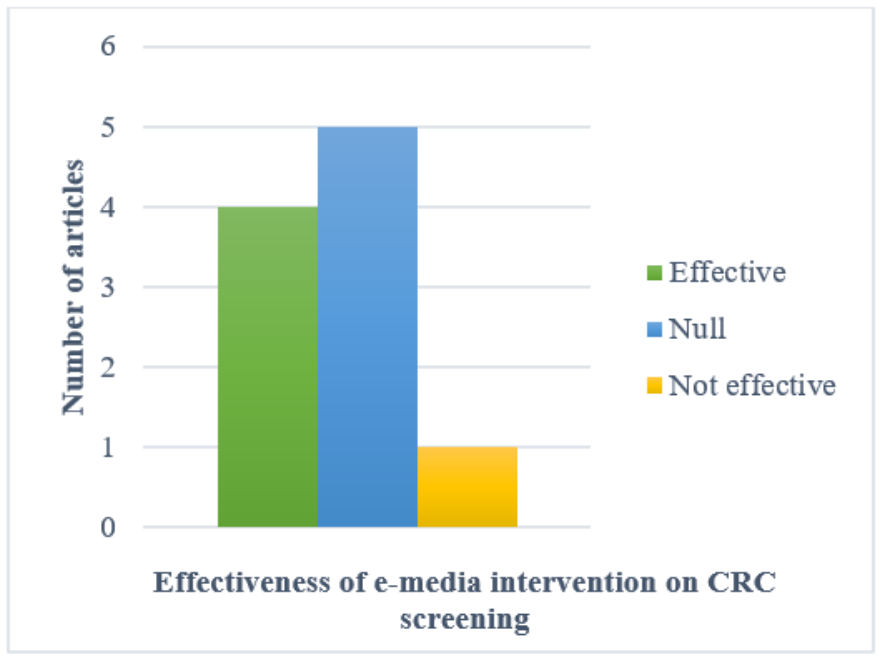

Figure 3. Comparison of effectiveness of e-media intervention on CRC screening $(N=10)$.

\subsubsection{Studies Included in Quantitative Synthesis}

Of the 10 studies included in this review, one [40] was not feasible for meta-analysis due to the lack of data. A total of 9145 samples were pooled from the nine feasible studies with either low or some risk of bias (Supplementary Tables S1 and S2, Supplementary Figure S2). The random-effects model revealed that there was a statistically significant positive effect of CRC-screening promotion using e-media interventions, where participants in the intervention groups were almost twice as likely to have CRC-screening completion $(\mathrm{OR}=1.62,95 \% \mathrm{CI}: 1.03-2.62, p<0.05)$ (Figure 4). Nonetheless, the clinical heterogeneity was high $\left(I^{2}=93 \%, p<0.05\right)$, and the funnel plot generated was asymmetrical (Figure 5). The sensitivity analysis results were no different after each trial had been excluded.

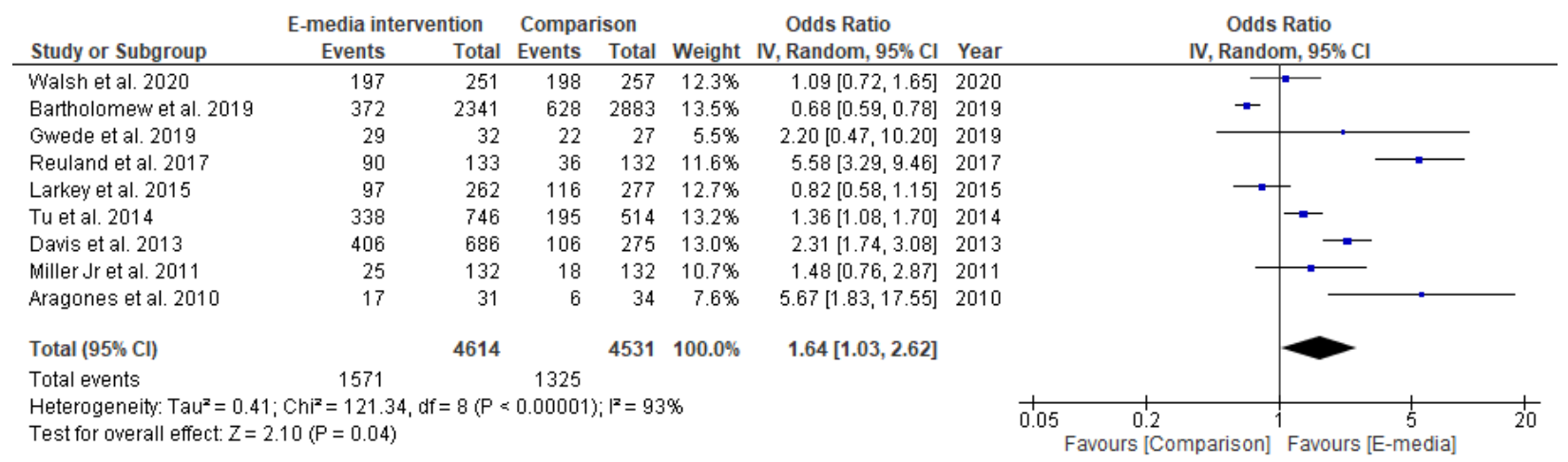

Figure 4. Random-effects forest plot for studies eligible for meta-analysis $(n=9)$. Box size represents study weighting. Diamond represents overall effect size and $95 \%$ CI.

In the subgroup analysis, two studies that used interactive programs as their emedia decision aid [31,37] reported a positive estimated pooled effect, wherein those in the intervention groups were more likely to complete their CRC screening $(\mathrm{OR}=1.19$, $95 \%$ CI 0.83-1.69, $p>0.05$ ); however, it did not reach statistical significance (Figure 6). On the other hand, the estimated pooled effect targeting the Latino population retrieved from two studies $[32,38]$ showed a significant positive effect of e-media interventions for CRC-screening promotion. In those studies, participants in the intervention groups were four times more likely to complete their screening compared to those in the comparison groups (OR $=4.06,95 \%$ CI 4.63-10.09, $p<0.05)$ (Figure 7). The analyses of heterogeneity in these two subgroups were able to prove homogeneous results $\left(I^{2}=0 \%, p<0.05\right)$. 


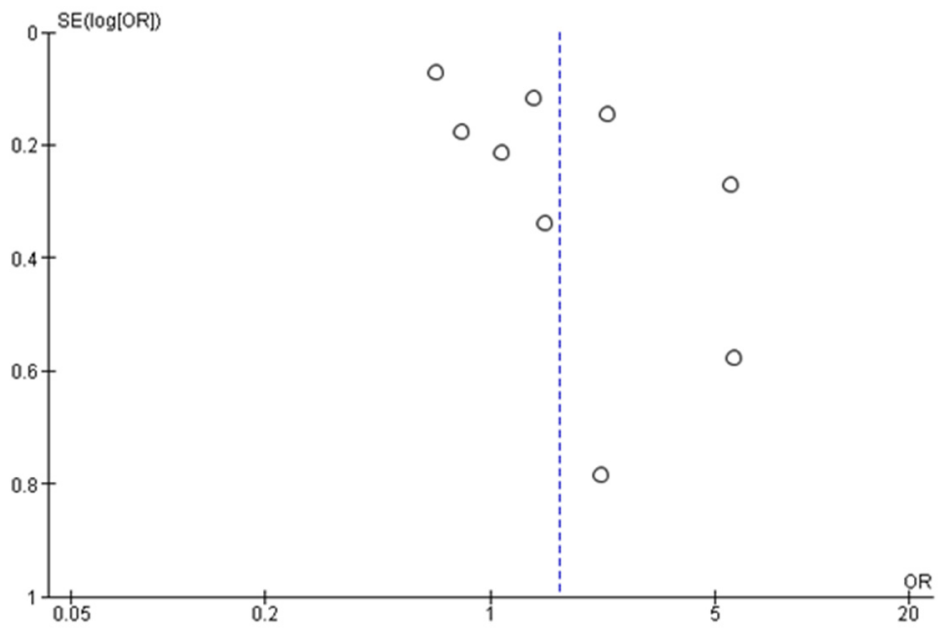

Figure 5. Funnel plot for studies eligible for meta-analysis $(n=9)$.

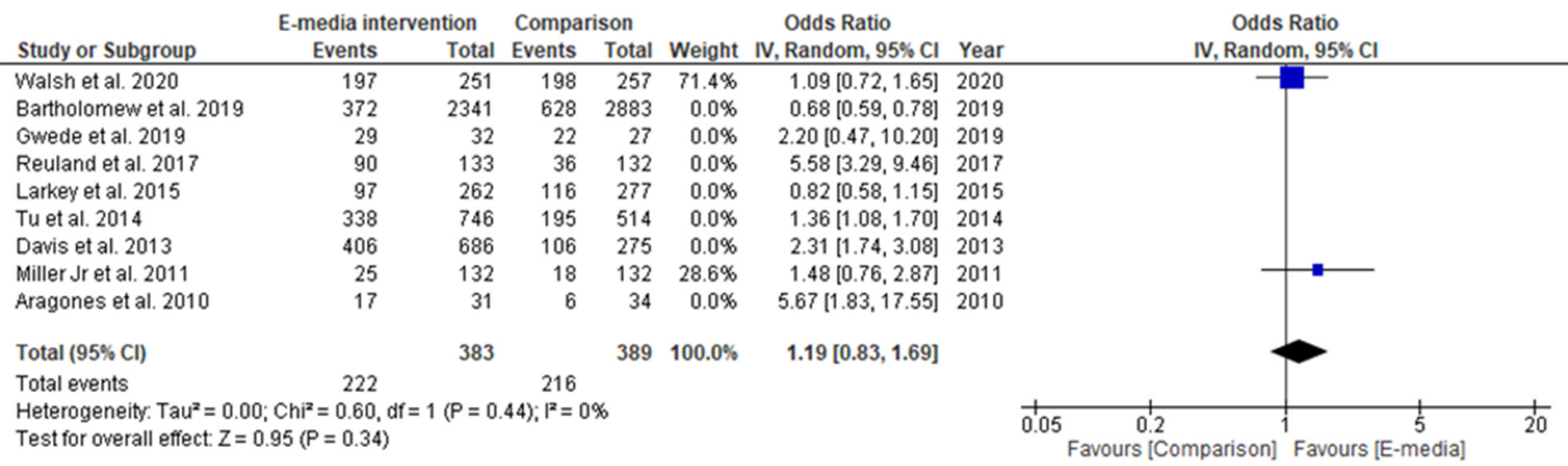

Figure 6. Random-effects forest plot for studies using interactive programs as intervention $(n=2)$. Box size represents study weighting. Diamond represents overall effect size and 95\% CI.

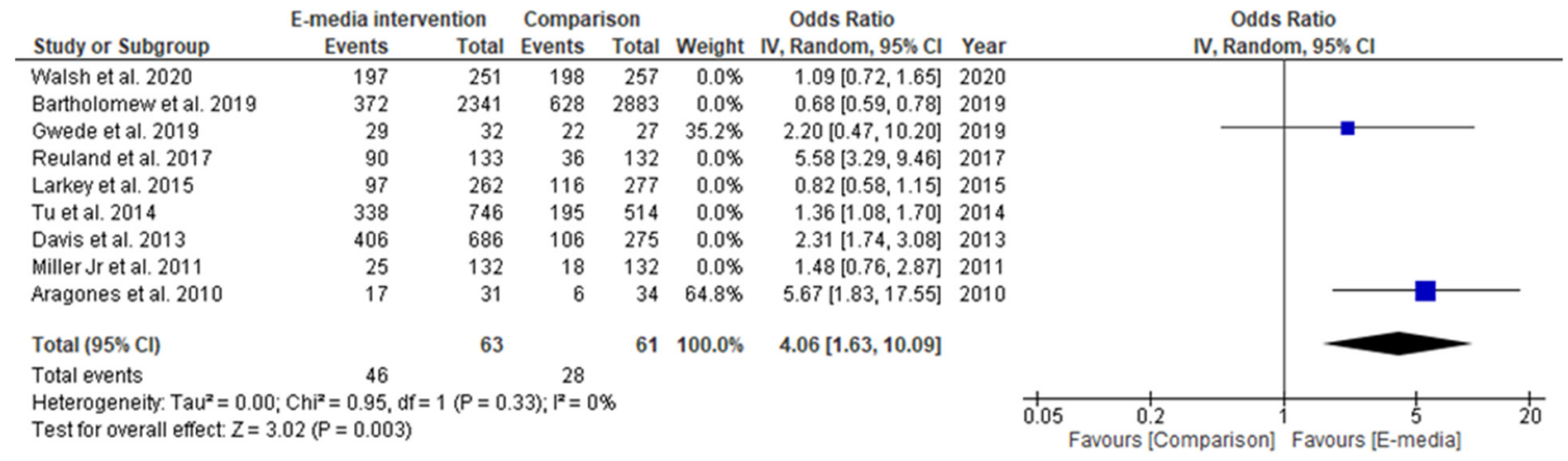

Figure 7. Random-effects forest plot for studies targeting Latino populations $(n=2)$. Box size represents study weighting. Diamond represents overall effect size and 95\% CI.

\subsubsection{Secondary Outcome}

The other main outcomes (Table 1) from the CRC-screening promotion interventions being reported are presented in Table 2, numbering a total of five secondary outcomes included in this review. Bartholomew et al. [39] reported evidence of a lower rate of spoiled FOBT kit return in the intervention groups than in the control groups in their two study populations ( $12.4 \%$ vs. $33.1 \%$ in Māori and $21.9 \%$ vs. $42.1 \%$ in a Pacific population). However, it did not reach statistical significance. Gwede et al. [32] found a significant association between CRC-screening promotion intervention with greater increases in CRC awareness 
and susceptibility, while cancer worry increased significantly in their comparison group. The ability to state CRC-screening test preference, readiness to receive CRC screening, and requesting CRC-screening tests were among the other main outcomes reported by Miller et al. [37], who found that intervention participants had significantly higher rates of CRC-screening preference ( $84 \%$ vs. $55 \%$ ) and readiness to receive screening ( $52 \%$ vs. $20 \%$ ) compared to their control counterparts. The rate of CRC-screening tests requested was also higher ( $30 \%$ vs. $21 \%)$; however, this was not significant. In general, these five secondary outcomes show the positive effects of CRC-screening interventions, regardless of their statistical significance values.

\section{Discussion}

This systematic literature review highlights the effectiveness of CRC-screening promotion practiced in PHC settings and research opportunities for improving CRC-screening participation for eligible and non-adherent patients in bowel cancer screening programs. This review makes a distinction between the types of e-media decision aid used for promoting CRC screening to optimize recruitment participation and follow-up, and towards better integration of CRC screening into existing PHC prevention pathways.

Our findings demonstrate that the rates of CRC-screening completion were higher among those who received e-media decision aid interventions in the majority of studies $(8 / 10)$, of which four reached statistical significance (Table 3). From the meta-analysis, it is evident that videos and interactive multimedia programs can significantly prompt previously unscreened people to choose their preferred screening test, thus resulting in higher rates of CRC-screening completion. This result also suggests that public populationbased CRC-screening intervention programs have been implemented heterogeneously across countries and regions, with significant CRC-screening completion rates seen in vulnerable Latino populations. The high clinical heterogeneity $\left(I^{2}=93 \%, p<0.05\right)$ shown may possibly reflects the variety of the population groups, sample sizes and decision aid contents including duration. In addition, the asymmetrical shape of funnel plot suggests for the existence of publication bias. This type of bias is induced by the fact that research with statistically significant results is more likely to be submitted and published than work with null or non-significant results. Thus, it poses a threat to the validity of such analyses. The implication of having publication bias may lead to an incorrect, usually over optimistic conclusion. Therefore, cautious interpretation is vital upon its existence. Finally, all secondary outcomes showed positive effects of CRC-screening promotion that uses emedia interventions when compared with their respective controls. Therefore, we conclude that e-media decision-aid interventions are effective for promoting CRC screening.

The effectiveness of health interventions has been linked to the use of health behavioral science theories. The health-belief model (HBM) has been applied most often for health concerns that are prevention-related and asymptomatic, such as early cancer detection, where beliefs are equally or more important than overt symptoms [41]. The HBM theorizes that people's beliefs about whether or not they are at risk for a disease, and their perceptions of the benefits of taking action to avoid it, influence their readiness to take action within the core construct of perceived susceptibility, perceived severity, perceived benefits, perceived barriers, cues to action, and self-efficacy [42]. These concepts have been explicitly applied in studies that showed effective results, where the videos and interactive multimedia programs used were able to inform participants of their perceived susceptibility by conveying the importance of screening, including CRC risk factors [33,34,38,40], persuade them to overcome perceived barriers [36], provide of cues to action by addressing the need to change their screening behavior [31] and prompt them towards self-efficacy to complete CRC screening [32,37].

While the HBM focuses on the individual, it also recognizes and addresses the social context in which health behaviours take place [43]. Thus, the infusion of the culturally tailored decision-aid concept also helps to enhance the positive effect of health interventions, as shown by some studies $[32,34,38]$ in the present review. This result is also congruent with the findings of other studies, where viewing culturally tailored decision aids can 
significantly increase patients' knowledge of CRC-screening recommendations and options, with a significant reduction in their decisional conflict and improved self-advocacy [44,45].

Through e-media platforms, not only can decision aids such as health-promotion videos exert great influence in patient decision-making, but they can also empower the willingness of many adults to use e-media to promote cancer screening to their peers [46]. Therefore, the studies in the present review should have adopted the Technology Acceptance Model [47] theory, along with the HBM, which highlights the central role of perceived usefulness, perceived ease-of-use, and users' acceptance constructs towards e-media or internet use [48]. Nonetheless, none of the included studies mentioned the theory or others of its kind. The constructs of such a theory are important, as health experts have concluded that there is a need for more comprehensive videos that are easily identifiable by patients [49]. This probably explains why we did not find many effective-cum-statistically significant interventions in this review.

Apart from the effective studies discussed above, two studies [35,39] reported lower rates of CRC-screening completion in their intervention groups. When comparing with other studies that had eligible patients as their study population, Bartholomew and colleagues [39] examined a population of those non-adherent to initial screening invitation. Knowing at the beginning of the study that the target population was non-adherent, the researchers should have applied all of the core constructs of the HBM theory, which the study lacked. In the second study that resulted in lower CRC-screening completion rate, Larkey and colleagues [35] used a video drama of "Papa" receiving CRC screening as the intervention. Reflecting on that study, there was a possible lack of the self-efficacy construct at the end of the story, and the participants merely perceived the video as a form of entertainment. Hence, decision aids that only hint at why screening interventions are given are not effective in comparison with the studies that were effective.

The literature search showed that the low uptake in CRC-screening reported in many parts of the world were more prominent among underserved populations such as the uninsured, recent immigrants, and in the most ethnically diverse areas, with a striking gradient according to socioeconomic status [50,51]. This probably explains why more studies on CRC-screening interventions in the present review focused on certain target populations (Table 2). Besides that, a majority of the studies in this review were conducted in the United States, hence possibly indicating its higher CRC burden than that in other countries and that its health authorities have taken the necessary health-promotion activities and research action.

E-media decision aids were often accompanied by other types of decision aids, such as patient navigator, brochure, pamphlet, or reminder letter, as shown in studies in the present review. In some other studies, multilayer-screening interventions involving patient navigators, such as nurses and other community health workers, were also used [52-55]. Of all the types of e-media decision-aids, videos were more frequently used compared with the other types, such as multimedia interactive programs or mobile phone reminders. The duration of decision aids used was 6-15 min, and was thought to be quite lengthy [56]; however, there was a significant association between video length and level of usefulness found [57].

Although we have commented on the positive effect, there were also some no-effect findings on the rate of CRC-screening completion among patients in PHC following videotype intervention. Zapka and colleagues discuss such results explicitly, where their studied samples were primarily middle-class white people who had high screening rates at baseline, and the trial was conducted during a period of increased health insurance coverage for lower endoscopy procedures with wide public media attention on CRC screening [58]. Similarly, studies conducted in groups with suboptimal CRC-screening rates reported no significant differences in the participants' attitudes, norms, or intentions regarding CRC-screening uptake [44,59].

These findings suggest that future theory-guided trial interventions are needed, as well as the need to examine health behavior moderators and mediators. Issues that are 
in line with the HBM theory that could explain negative health behavior may also be considered in developing CRC-screening decision aids [60]; for example, the narration of personal experiences of cancer survivors could provide many cues to action for those who have never encountered this potentially deadly but preventable disease, and thus should be incorporated into health promotion activities.

\section{Limitation}

Reporting and publication bias may have affected our findings. The independent effect of multicomponent elements of e-media decision-aid interventions was often uncertain, as can be inferred from the high heterogeneity in meta-analysis. Despite the limitations, the findings are relevant to settings in private practice or through an organized screening program, given the role of PHC services for preventive care follow-up. To our knowledge, this is the first review that focuses on e-media decision aids in the CRC-screening pathway.

\section{Conclusions}

Overall, this review provides effective evidence of intervention studies that used e-media platforms for promoting CRC screening. In other words, e-media decision aids have been proven to have great potential for increasing CRC-screening participation in the era of rapid electronic gadget revolution in combination with the Internet of Things. If integrated appropriately in PHC settings, these interventions could act as an effective learning interface for patients with CRC and their families. Therefore, development and application of culturally tailored e-media decision aids aimed at increasing CRC-screening completion should be prioritized. However, healthcare providers and organizations must be aware of the limitations and pitfalls of these platforms and must address them appropriately.

Supplementary Materials: The following are available online at https:/ / www.mdpi.com/article/10 .3390 /ijerph18158190/s1. Figure S1: Search strategy or the medical subject heading (MeSH) keywords used. Figure S2: Risk of bias assessment according to domains using the Cochrane's risk-of-bias tool for randomized trials (RoB 2), and risk-of-bias tool for cluster-randomized trials (RoB 2 CRT). Total number of study, $N=10$. Table S1: Risk of bias assessment according to the Cochrane's risk-of-bias tools for all articles in this review. Table S2: Risk of bias assessment according to domains using the Cochrane's risk-of-bias tool for randomized trials (RoB 2), and risk-of-bias tool for cluster-randomized trials (RoB 2 CRT). Table S3: Sources of funding for all articles in this review.

Author Contributions: Conceptualization N.S.R. and M.I.I.; methodology N.S.R., A.M.N. and M.R.H.; formal analysis N.S.R., A.M.N. and M.R.H.; resources A.M.N. and M.R.H.; writing N.S.R. and A.M.N.; visualization M.I.I.; supervision A.M.N.; project administration M.R.A.M. All authors have read and agreed to the published version of the manuscript.

Funding: This research received no external funding.

Institutional Review Board Statement: The review was part of a clinical research approved by the Medical Research Ethics Committee of UKM (protocol code FF-2020-155 and date of approval 20 April 2020).

Data Availability Statement: The data presented in this study are available in this article.

Acknowledgments: We would like to thank the Universiti Kebangsaan Malaysia for its support and technical guidance in conducting this study.

Conflicts of Interest: The authors declare no conflict of interest.

\section{References}

1. Bray, F.; Ferlay, J.; Soerjomataram, I.; Siegel, R.L.; Torre, L.A.; Jemal, A. Global cancer statistics 2018: GLOBOCAN estimates of incidence and mortality worldwide for 36 cancers in 185 countries. CA Cancer J. Clin. 2018, 68, 394-424. [CrossRef] [PubMed]

2. Global Cancer Observatory. Estimated Age-Standardized Incidence Rates (World) in 2020, Worldwide, All Ages. Available online: https://gco.iarc.fr/today / online-analysis-multi-bars?v=2020\&mode=cancer\&mode_population=countries\& population $=900 \&$ populations $=900 \&$ key $=$ asr $\&$ sex $=1 \&$ cancer $=39 \&$ type $=0 \&$ statistic $=5 \&$ prevalence $=0 \&$ population $\_$group $=0 \&$ ages_group $\% 5 \mathrm{~B} \% 5 \mathrm{D}=0$ \&ages_group $\% 5 \mathrm{~B} \% 5 \mathrm{D}=17 \&$ nb_items $=10$ \&group_cancer $=1$ \&include_nmsc $=1 \&$ include_nmsc_other=1\& 
type_multiple $=\% 257 \mathrm{~B} \% 2522$ inc $\% 2522 \% 253 \mathrm{Atrue} \% 252 \mathrm{C} \% 2522 \mathrm{mort} \% 2522 \% 253 \mathrm{Afalse} \% 252 \mathrm{C} \% 2522 \mathrm{prev} \% 2522 \% 253 \mathrm{Afalse} \% 25$ 7D\&orientation=horizontal\&type_sort=0\&type_nb_items=\%257B $\% 2522$ top $\% 2522 \% 253 \mathrm{Atrue} \% 252 \mathrm{C} \% 2522 \mathrm{bottom} \% 2522 \% 253$ Afalse\%257D (accessed on 15 December 2020).

3. Wong, M.C.; Huang, J.; Lok, V.; Wang, J.; Fung, F.; Ding, H.; Zheng, Z.-J. Differences in incidence and mortality trends of colorectal cancer, worldwide, based on sex, age, and anatomic location. Clin. Gastroenterol. Hepatol. 2021, 19, 955-966.e61. [CrossRef] [PubMed]

4. Keum, N.; Giovannucci, E. Global burden of colorectal cancer: Emerging trends, risk factors and prevention strategies. Nat. Rev. Gastroenterol. Hepatol. 2019, 16, 713-732. [CrossRef] [PubMed]

5. Azizah, A.M.; Hashimah, B.; Nirmal, K.; Siti Zubaidah, A.R.; Puteri, N.A.; Nabihah, A.; Sukumaran, R.; Balqis, B.; Nadia, S.M.R.; Sharifah, S.S.S.; et al. Malaysia National Cancer Registry Report (MNCRR) 2012-2016; Ministry of Health Malaysia: Putrajaya, Malaysia, 2019.

6. Douaiher, J.; Ravipati, A.; Grams, B.; Chowdhury, S.; Alatise, O.; Are, C. Colorectal cancer-global burden, trends, and geographical variations. J. Surg. Oncol. 2017, 115, 619-630. [CrossRef] [PubMed]

7. Walter, F.M.; Emery, J.D.; Mendonca, S.; Hall, N.; Morris, H.C.; Mills, K.; Dobson, C.; Bankhead, C.; Johnson, M.; Abel, G.A. Symptoms and patient factors associated with longer time to diagnosis for colorectal cancer: Results from a prospective cohort study. Br. J. Cancer 2016, 115, 533-541. [CrossRef]

8. Wolf, A.M.; Fontham, E.T.; Church, T.R.; Flowers, C.R.; Guerra, C.E.; LaMonte, S.J.; Etzioni, R.; McKenna, M.T.; Oeffinger, K.C.; Shih, Y.C.T. Colorectal cancer screening for average-risk adults: 2018 guideline update from the American Cancer Society. $C A$ Cancer J. Clin. 2018, 68, 250-281. [CrossRef]

9. Schreuders, E.H.; Ruco, A.; Rabeneck, L.; Schoen, R.E.; Sung, J.J.; Young, G.P.; Kuipers, E.J. Colorectal cancer screening: A global overview of existing programmes. Gut 2015, 64, 1637-1649. [CrossRef]

10. Honein-AbouHaidar, G.N.; Kastner, M.; Vuong, V.; Perrier, L.; Daly, C.; Rabeneck, L.; Straus, S.; Baxter, N.N. Systematic review and meta-study synthesis of qualitative studies evaluating facilitators and barriers to participation in colorectal cancer screening. Cancer Epidemiol. Prev. Biomark. 2016, 25, 907-917. [CrossRef]

11. Chen, H.; Li, N.; Ren, J.; Feng, X.; Lyu, Z.; Wei, L.; Li, X.; Guo, L.; Zheng, Z.; Zou, S. Participation and yield of a population-based colorectal cancer screening programme in China. Gut 2019, 68, 1450-1457. [CrossRef]

12. Norwati, D.; Harmy, M.; Norhayati, M.; Amry, A. Colorectal cancer screening practices of primary care providers: Results of a national survey in Malaysia. Asian Pac. J. Cancer Prev. 2014, 15, 2901. [CrossRef]

13. May, F.P.; Almario, C.V.; Ponce, N.; Spiegel, B.M. Racial minorities are more likely than whites to report lack of provider recommendation for colon cancer screening. Am. J. Gastroenterol. 2015, 110, 1388-1394. [CrossRef]

14. Ooi, C.Y.; Hanafi, N.S.; Liew, S.M. Knowledge and practice of colorectal cancer screening in an urban setting: Cross-sectional survey of primary care physicians in government clinics in Malaysia. Singap. Med. J. 2019, 60, 596. [CrossRef]

15. Gandomani, H.S.; Aghajani, M.; Mohammadian-Hafshejani, A.; Tarazoj, A.A.; Pouyesh, V.; Salehiniya, H. Colorectal cancer in the world: Incidence, mortality and risk factors. Biomed. Res. Ther. 2017, 4, 1656-1675. [CrossRef]

16. Abu Hassan, M.R.; Nik Mustapha, N.R.; Ahmad, F.; Soelar, S.A.; Mohd Suan, M.A.; Ismail, I.; Syahireen Mohammed, S.R.N.; Ali, S.M.; Chan, H.-K. National Cancer Patient Registry—Colorectal Cancer: Report for the Northern Region of Malaysia (2008-2014); Ministry of Health Malaysia, Clinical Research Centre: Alor Setar, Kedah, Malaysia, 2017.

17. Gough, A.; Hunter, R.F.; Ajao, O.; Jurek, A.; McKeown, G.; Hong, J.; Barrett, E.; Ferguson, M.; McElwee, G.; McCarthy, M. Tweet for behavior change: Using social media for the dissemination of public health messages. JMIR Public Health Surveill. 2017, 3, e14. [CrossRef]

18. Rowsell, A.; Muller, I.; Murray, E.; Little, P.; Byrne, C.D.; Ganahl, K.; Müller, G.; Gibney, S.; Lyles, C.R.; Lucas, A. Views of people with high and low levels of health literacy about a digital intervention to promote physical activity for diabetes: A qualitative study in five countries. J. Med. Internet Res. 2015, 17, e230. [CrossRef] [PubMed]

19. Chou, W.-y.S.; Prestin, A.; Lyons, C.; Wen, K.-Y. Web 2.0 for health promotion: Reviewing the current evidence. Am. J. Public Health 2013, 103, e9-e18. [CrossRef]

20. Hoffman, A.S.; Volk, R.J.; Saarimaki, A.; Stirling, C.; Li, L.C.; Härter, M.; Kamath, G.R.; Llewellyn-Thomas, H. Delivering patient decision aids on the Internet: Definitions, theories, current evidence, and emerging research areas. BMC Med. Inform. Decis. Mak. 2013, 13, S13. [CrossRef]

21. O'Mara, B. Social media, digital video and health promotion in a culturally and linguistically diverse Australia. Health Promot. Int. 2013, 28, 466-476. [CrossRef] [PubMed]

22. Lupton, D. Health promotion in the digital era: A critical commentary. Health Promot. Int. 2014, 30, 174-183. [CrossRef]

23. Resources, P. MEDLINE®: Description of the Database. Available online: https://www.nlm.nih.gov/bsd/medline.html (accessed on 15 December 2020).

24. Uzzi, B.; K.S.o.M.N.U. Web of Science. Available online: https:// clarivate-com.ezplib.ukm.my/webofsciencegroup (accessed on 15 December 2020).

25. Cochrane Library. About the Cochrane Library. Available online: https://www.cochranelibrary.com/about/about-cochranelibrary (accessed on 15 December 2020).

26. Fu, H.; Wang, M.; Li, P.; Jiang, S.; Hu, W.; Guo, X.; Cao, M. Tracing knowledge development trajectories of the internet of things domain: A main path analysis. IEEE Trans. Ind. Informatics 2019, 15, 6531-6540. [CrossRef] 
27. Moher, D.; Liberati, A.; Tetzlaff, J.; Altman, D.G.; The PRISMA Group. Preferred reporting items for systematic reviews and meta-analyses: The PRISMA statement. PLoS Med. 2009, 6, e1000097. [CrossRef] [PubMed]

28. Holden, C.A.; Frank, O.; Caruso, J.; Turnbull, D.; Reed, R.L.; Miller, C.L.; Olver, I. From participation to diagnostic assessment: A systematic scoping review of the role of the primary healthcare sector in the National Bowel Cancer Screening Program. Aust. J. Prim. Health 2020, 26, 191-206. [CrossRef]

29. Sterne, J.A.; Savović, J.; Page, M.J.; Elbers, R.G.; Blencowe, N.S.; Boutron, I.; Cates, C.J.; Cheng, H.-Y.; Corbett, M.S.; Eldridge, S.M. RoB 2: A revised tool for assessing risk of bias in randomised trials. BMJ 2019, 366, 14898. [CrossRef]

30. RevMan. Review Manager (RevMan) [Computer Program]. Available online: https://training.cochrane.org/online-learning/ core-software-cochrane-reviews/revman/revman-5-download (accessed on 20 December 2020).

31. Walsh, J.; Potter, M.; Salazar, R.; Ozer, E.; Gildengorin, G.; Dass, N.; Green, L. PreView: A randomized trial of a multi-site intervention in diverse primary care to increase rates of age-appropriate cancer screening. J. Gen. Intern. Med. 2020, 35, 449-456. [CrossRef]

32. Gwede, C.K.; Sutton, S.K.; Chavarria, E.A.; Gutierrez, L.; Abdulla, R.; Christy, S.M.; Lopez, D.; Sanchez, J.; Meade, C.D. A culturally and linguistically salient pilot intervention to promote colorectal cancer screening among Latinos receiving care in a Federally Qualified Health Center. Health Educ. Res. 2019, 34, 310-320. [CrossRef] [PubMed]

33. Reuland, D.S.; Brenner, A.T.; Hoffman, R.; McWilliams, A.; Rhyne, R.L.; Getrich, C.; Tapp, H.; Weaver, M.A.; Callan, D.; Cubillos, L. Effect of combined patient decision aid and patient navigation vs usual care for colorectal cancer screening in a vulnerable patient population: A randomized clinical trial. JAMA Intern. Med. 2017, 177, 967-974. [CrossRef] [PubMed]

34. Tu, S.-P.; Chun, A.; Yasui, Y.; Kuniyuki, A.; Yip, M.-P.; Taylor, V.; Bastani, R. Adaptation of an evidence-based intervention to promote colorectal cancer screening: A quasi-experimental study. Implement. Sci. 2014, 9, 85. [CrossRef]

35. Larkey, L.K.; McClain, D.; Roe, D.J.; Hector, R.D.; Lopez, A.M.; Sillanpaa, B.; Gonzalez, J. Randomized controlled trial of storytelling compared to a personal risk tool intervention on colorectal cancer screening in low-income patients. Am. J. Health Prom. 2015, 30, e59-e70. [CrossRef] [PubMed]

36. Davis, T.; Arnold, C.; Rademaker, A.; Bennett, C.; Bailey, S.; Platt, D.; Reynolds, C.; Liu, D.; Carias, E.; Bass, P., III; et al. Improving colon cancer screening in community clinics. Cancer 2013, 119, 3879-3886. [CrossRef] [PubMed]

37. Miller, D.P., Jr.; Spangler, J.G.; Case, L.D.; Goff, D.C., Jr.; Singh, S.; Pignone, M.P. Effectiveness of a web-based colorectal cancer screening patient decision aid: A randomized controlled trial in a mixed-literacy population. Am. J. Prev. Med. 2011, 40, 608-615. [CrossRef]

38. Aragones, A.; Schwartz, M.D.; Shah, N.R.; Gany, F.M. A randomized controlled trial of a multilevel intervention to increase colorectal cancer screening among Latino immigrants in a primary care facility. J. Gen. Intern. Med. 2010, 25, 564-567. [CrossRef]

39. Bartholomew, K.; Zhou, L.; Crengle, S.; Buswell, E.; Buckley, A.; Sandiford, P. A targeted promotional DVD fails to improve Māori and Pacific participation rates in the New Zealand bowel screening pilot: Results from a pseudo-randomised controlled trial. BMC Publ. Health 2019, 19, 1245. [CrossRef] [PubMed]

40. Besharati, F.; Karimi-Shahanjarini, A.; Hazavehei, S.M.M.; Bashirian, S.; Bagheri, F.; Faradmal, J. Development of a colorectal cancer screening intervention for Iranian adults: Appling intervention mapping. Asian Pac. J. Cancer Prev. 2017, $18,2193$. [PubMed]

41. Glanz, K.; Bishop, D.B. The role of behavioral science theory in development and implementation of public health interventions. Annu. Rev. Public Health 2010, 31, 399-418. [CrossRef]

42. Skinner, C.S.; Tiro, J.; Champion, V.L. Background on the health belief model. Health Behav. Theory Res. Pract. $2015,75$.

43. Green, E.C.; Murphy, E.M.; Gryboski, K. The health belief model. Wiley Encycl. Health Psychol. 2020, 211-214. [CrossRef]

44. Hoffman, A.S.; Lowenstein, L.M.; Kamath, G.R.; Housten, A.J.; Leal, V.B.; Linder, S.K.; Jibaja-Weiss, M.L.; Raju, G.S.; Volk, R.J. An entertainment-education colorectal cancer screening decision aid for African American patients: A randomized controlled trial. Cancer 2017, 123, 1401-1408. [CrossRef]

45. Volk, R.J.; Linder, S.K.; Lopez-Olivo, M.A.; Kamath, G.R.; Reuland, D.S.; Saraykar, S.S.; Leal, V.B.; Pignone, M.P. Patient decision aids for colorectal cancer screening: A systematic review and meta-analysis. Am. J. Prev. Med. 2016, 51, 779-791. [CrossRef]

46. Cutrona, S.L.; Roblin, D.W.; Wagner, J.L.; Gaglio, B.; Williams, A.E.; Stone, R.T.; Field, T.S.; Mazor, K.M. Adult willingness to use email and social media for peer-to-peer cancer screening communication: Quantitative interview study. JMIR Res. Protoc. 2013, 2, e52. [CrossRef]

47. Merino, P.; Bustamante, E.; Campillo-Artero, C.; Bartual, E.; Tuero, G.; Marí, J. Patient safety certification in a Department of Intensive Care Medicine: Our experience with standard UNE 179003:2013. Med. Intensiv. 2014, 38, 297-304. [CrossRef]

48. Davis, F.D. Perceived usefulness, perceived ease of use, and user acceptance of information technology. MIS Q. 1989, 13, 319-340. [CrossRef]

49. Mukewar, S.; Mani, P.; Wu, X.; Lopez, R.; Shen, B. YouTube®and inflammatory bowel disease. J. Crohns Colitis 2013, 7, 392-402. [CrossRef]

50. Gupta, S.; Sussman, D.A.; Doubeni, C.A.; Anderson, D.S.; Day, L.; Deshpande, A.R.; Joseph Elmunzer, B.; Laiyemo, A.O.; Mendez, J.; Somsouk, M. Challenges and possible solutions to colorectal cancer screening for the underserved. J. Natl. Cancer Inst. 2014, 106, dju032. [CrossRef] 
51. Von Wagner, C.; Baio, G.; Raine, R.; Snowball, J.; Morris, S.; Atkin, W.; Obichere, A.; Handley, G.; Logan, R.F.; Rainbow, S. Inequalities in participation in an organized national colorectal cancer screening programme: Results from the first 2.6 million invitations in England. Int. J. Epidemiol. 2011, 40, 712-718. [CrossRef]

52. Escoffery, C.; Fernandez, M.E.; Vernon, S.W.; Liang, S.; Maxwell, A.E.; Allen, J.D.; Dwyer, A.; Hannon, P.A.; Kohn, M.; DeGroff, A. Patient navigation in a colorectal cancer screening program. J Public Health Manag Pract. 2015, 21, 433. [CrossRef]

53. Kemper, K.E.; Glaze, B.L.; Eastman, C.L.; Waldron, R.C.; Hoover, S.; Flagg, T.R.; Tangka, F.K.; Subramanian, S. Effectiveness and cost of multilayered colorectal cancer screening promotion interventions at federally qualified health centers in Washington State. Cancer 2018, 124, 4121-4129. [CrossRef]

54. Roland, K.B.; Milliken, E.L.; Rohan, E.A.; DeGroff, A.; White, S.; Melillo, S.; Rorie, W.E.; Signes, C.C.; Young, P.A. Use of community health workers and patient navigators to improve cancer outcomes among patients served by federally qualified health centers: A systematic literature review. Health Equity 2017, 1, 61-76. [CrossRef] [PubMed]

55. Rohan, E.A.; Slotman, B.; DeGroff, A.; Morrissey, K.G.; Murillo, J.; Schroy, P. Refining the patient navigation role in a colorectal cancer screening program: Results from an intervention study. J. Natl. Compr. Cancer Netw. 2016, 14, 1371-1378. [CrossRef] [PubMed]

56. Sahin, A.N.; Sahin, A.S.; Schwenter, F.; Sebajang, H. YouTube videos as a source of information on colorectal cancer: What do our patients learn? J. Cancer Educ. 2019, 34, 1160-1166. [CrossRef] [PubMed]

57. Brar, J.; Ferdous, M.; Abedin, T.; Turin, T.C. Online Information for Colorectal Cancer Screening: A Content Analysis of YouTube Videos. J. Cancer Educ. 2020, 35, 1-6. [CrossRef] [PubMed]

58. Zapka, J.G.; Lemon, S.C.; Puleo, E.; Estabrook, B.; Luckmann, R.; Erban, S. Patient education for colon cancer screening: A randomized trial of a video mailed before a physical examination. Ann. Intern. Med. 2004, 141, 683-692. [CrossRef] [PubMed]

59. Gabel, P.; Edwards, A.; Kirkegaard, P.; Larsen, M.B.; Andersen, B. The LEAD trial-The effectiveness of a decision aid on decision making among citizens with lower educational attainment who have not participated in FIT-based colorectal cancer screening in Denmark: A randomised controlled trial. Patient Educ. Couns. 2020, 103, 359-368. [CrossRef] [PubMed]

60. Farooqui, M.; Hassali, M.A.; Knight, A.; Shafie, A.A.; Farooqui, M.A.; Saleem, F.; Haq, N.-u.; Aljadhey, H. A qualitative exploration of Malaysian cancer patients' perceptions of cancer screening. BMC Public Health 2013, 13, 48. [CrossRef] [PubMed] 